\title{
Decreased Sensory Stimulation Reduces Behavioral Responding, Retards Development, and Alters Neuronal Connectivity in Caenorhabditis elegans
}

\author{
Jacqueline K. Rose, ${ }^{1,2 \star}$ Susan Sangha, ${ }^{1,2 \star}$ Susan Rai, ${ }^{1,2 \star}$ Kenneth R. Norman, ${ }^{3}$ and Catharine H. Rankin ${ }^{1,2}$ \\ ${ }^{1}$ Department of Psychology, ${ }^{2}$ Brain Research Centre, and ${ }^{3}$ Department of Zoology, University of British Columbia, Vancouver, British Columbia, Canada \\ V6T 1 Z4
}

\begin{abstract}
Activity-dependent plasticity is a critical component of nervous systems. We show that in Caenorhabditis elegans, worms raised in isolation made smaller responses to mechanosensory stimulation and were smaller and slower to begin laying eggs than age-matched group-raised worms. The glutamate receptor gene GLR-1 was critical for the observed alterations in behavior but not in size, whereas the cGMP-dependent protein kinase gene EGL-4 was critical for the observed changes in size but not the changes in behavior. Mechanosensory stimulation during development reversed the effects of isolation on behavior and began to reduce the effects of isolation on size. In C. elegans, the six mechanosensory touch neurons synapse onto the four pair of command interneurons for forward and backward movement. Touch (mechanosensory) neurons of worms raised in isolation expressed lower levels of green fluorescent protein (GFP)tagged synaptobrevin than touch neurons of worms raised in colonies. Command interneurons of worms raised in isolation expressed lower levels of GFP-tagged glutamate receptors than command interneurons of worms raised in groups. Brief mechanical stimulation during larval development rescued the expression of GFP-tagged glutamate receptors but not GFP-tagged synaptobrevin. Together, these results indicate that the level of stimulation experienced by $C$. elegans during development profoundly affects the development of neuronal connectivity and has widespread cellular and behavioral consequences.
\end{abstract}

Key words: early deprivation; enrichment; activity dependent; glutamate; model system; development

\section{Introduction}

A fundamental characteristic of developing nervous systems is that they are plastic, shaped in part by their own activity (Bonhoeffer and Shatz, 1998). There is strong evidence that during development, activity-dependent processes sculpt the final patterns and strengths of synaptic connections in all nervous systems. Studies have shown that rats raised in isolation, with minimal stimulation, have thinner visual cortices (Diamond et al., 1966), less dendritic material in visual cortical neurons (Volkmar and Greenough, 1972), and fewer synapses per neuron (Turner and Greenough, 1985) than rats raised in enriched environments. Male zebra finches reared in isolation show alterations in the

\footnotetext{
Received Jan. 11, 2005; revised June 21, 2005; accepted June 22, 2005.

This work was supported by Natural Sciences and Engineering Research Council of Canada (NSERC) and Canadian Institutes of Health Research (CIHR) operating grants to C.H.R. and by NSERC Postgraduate Scholarship B, the Michael Smith Foundation for Health Research, and CIHR fellowships to J.K.R. In addition, we gratefully acknowledge the British Columbia Ministry of Children and Family Development through the Human Early Learning Partnership (HELP). The views presented in the paper are solely those of the authors and do not represent the policy of HELP or the province. We thank Anita Gock, Harjit Aulakh, Jennifer Jasper, and Susan Rai for running or piloting some of the experiments and Amelia Wan, Lianne Tang, and Stephan Steidl for scoring and helping with some of the experiments. We thank Josh Kaplan for GLR-1::GFP worms, Michael Nonet for ${ }_{p}$ mec-7::SNB-1::GFP worms, and Ann Hart for kp537 g/r-1 rescue worms as well as the Caenorhabditis Genetics Center (St. Paul, MN) for other strains of worms used in these studies.

*J.K.R., S.S., and S.R. contributed equally to this work.

Correspondence should be addressed to Catharine Rankin, Department of Psychology, University of British Columbia, 2136 West Mall, Vancouver, British Columbia, Canada V6T 1Z4. E-mail: crankin@psych.ubc.ca. DOI:10.1523/JNEUROSCI.1833-05.2005

Copyright $\odot 2005$ Society for Neuroscience $\quad$ 0270-6474/05/257159-10\$15.00/0
}

shape and number of spines on one type of neuron (Rollenhagen and Bischof, 1994). In addition to these findings in vertebrate organisms, invertebrates can also show the effects of activity on development. For example, Drosophila melanogaster reared in isolation showed $15 \%$ fewer Kenyon cell fibers in their mushroom bodies than flies raised in group conditions (Heisenberg et al., 1995).

The difficulties in studying the effects of varying sensory stimulation during development in vertebrates include the size and complexity of the vertebrate nervous system and the length of the developmental period. Here, we propose the nematode Caenorhabditis elegans as a model system in which to study the effects of varying sensory stimulation on the developing nervous system. $C$. elegans has a short generation time, reaching adulthood in $<4 \mathrm{~d}$. In addition, the nervous system of $C$. elegans is composed of only 302 neurons. The number and types of neurons as well as the connections between them have been proposed to be mostly invariant between individuals, and synaptic connections are highly characteristic and axon morphology predictable (White et al., 1986). Approximately $80-90 \%$ of the synapses appear to be reproducible between individuals (the remaining synapses are widely scattered and irreproducible from one animal to the next) (Hall and Russell, 1991). This might suggest that the nervous system of C. elegans is predetermined and not sensitive to activity. However, studies of genetic mutations that alter neuronal function suggest that normal activity might be necessary for normal 
axonal branching and morphology as well as normal growth in $C$. elegans (Peckol et al., 1999; Schaefer et al., 2000, Fujiwara et al., 2002). In addition, a number of studies have shown plasticity of the tap withdrawal response because adult $C$. elegans display a variety of forms of learning and memory (Rankin et al., 1990) (for review, see Rose and Rankin, 2001).

In the laboratory, groups of $C$. elegans grow on small agarfilled Petri plates and feed on Escherichia coli (Brenner, 1974). To test whether mechanosensory stimulation from other worms influences the development of the nervous system, wild-type and mutant strains of worms were raised either with conspecifics (colony-raised) or in isolation (isolate-raised), and then the response to a mechanosensory stimulus, size, egg-laying, and expression of green fluorescent protein (GFP) for presynaptic and postsynaptic markers were compared in the two groups.

\section{Materials and Methods \\ Subjects}

All of the measurements were made on 4-d-old adult C. elegans. A total of 2814 C. elegans were used in these experiments. Of these, 2301 were wild-type N2. To assess the role of chemosensory cues in the effects of isolation, 40 osm-6(p811) worms were used. In this strain, the mutation results in disrupted structure of sensory cilia that causes defects in all types of chemosensory behaviors (Dusenbery and Barr, 1980; Peckol et al., 1999). To assess the role of $g l r-1$ in the effects of isolation 124 of $g l r-1(\mathrm{n} 2461)$ and 157 of the $g l r-1$, rescue strain kp537 was used (in these experiments, $n$ values were high because the rescue strain is not integrated and responses were more variable than in wild-type worms). To assess the role of mec-4(e1611) and egl-4(n477) in the effects of isolation, $37 \mathrm{mec}-4$ worms and $42 \mathrm{egl}-4$ worms were reared in either isolation or colonies and tested. For confocal imaging, two genetically engineered strains were used: GLR-1::GFP and $m e c-7::$ SNB-1::GFP. GLR-1::GFP worms $(n=65)$ contain chimeric receptors made up of GLR-1 (a homolog of a kainate/AMPA-type glutamate receptor expressed on the interneurons of the tap withdrawal circuit) tagged with GFP, which has been used to visualize synapses in C. elegans (Rongo and Kaplan, 1999). In ${ }_{\mathrm{p}}$ mec-7::SNB-1::GFP $(n=48)$, we looked at GFP expression of the gene for C. elegans synaptobrevin (snb-1), a protein associated with synaptic vesicles that plays a role in regulating vesicle fusion at the synaptic terminal. SNB-1::GFP was expressed under the control of the mec-7 promoter, which targets this GFP expression to the six mechanosensory neurons of the tap withdrawal circuit (Nonet, 1999).

\section{Procedure and apparatus}

All plates were $60 \mathrm{~mm}$ in diameter and $15 \mathrm{~mm}$ deep, filled with $10 \mathrm{ml}$ of nematode growth medium agar, and streaked with one to two drops of $E$. coli (strain OP50) to produce a circular lawn $\sim 10 \mathrm{~mm}$ in diameter (Brenner, 1974). For behavioral testing, a platinum wire pick was used to transfer adult worms (96-100 h after eggs were laid) from the plates where they were grown to a test plate without any E. coli.

The test plate, in a plate holder attached to a micromanipulator, rested on the stage of the stereomicroscope (Wild M3Z; Leica, Nussloch, Germany). A video camera (Digital 5100; Panasonic, Secaucus, NJ) was attached to the stereomicroscope and also to a video cassette recorder (Panasonic AG1960) and television monitor (PM-1271A; NEC, Tokyo, Japan). The date and time were displayed on the monitor via a time-date generator (Panasonic WJ-810) and videotaped. After 2 unrecorded minutes, the stage lamp was turned on, and a single tap was delivered to the side of the plate via a copper rod driven by a Grass Instruments (Quincy, MA) S88 stimulator.

\section{Rearing condition}

Colony-raised worms were produced by allowing five adult hermaphrodites to lay between 30 and 40 eggs on a Petri plate with E. coli, then removing the adults so that only the eggs remained. Isolate-raised plates were produced by allowing one adult hermaphrodite to lay one egg on a Petri plate with E. coli, then removing the adult and any extra eggs so that only one egg remained. To protect worms from extraneous stimulation, all plates were then placed in foam- or cotton-lined boxes and placed in a $20 \pm 0.2^{\circ} \mathrm{C}$ incubator. Plates were not touched until testing $(96-100 \mathrm{~h}$ later).

\section{Conditioned plates}

In one experiment, isolate and colony worms were raised on plates that had contained colonies of worms for $4 \mathrm{~d}$ before the experiment. All of these "conditioning" worms were removed from the plates before the egg-laying for the experiment. This was done to provide the chemical stimuli of groups of worms for the isolate-raised worms.

\section{Larval stimulation}

In experiments with a stimulated isolate-raised condition, worms were treated as described above for the isolate-raised and colony-raised conditions; however, in the third larval stage of development ("L3"; 36 h of age), they were given a brief period of mechanosensory stimulation. (L3 was chosen as the approximate mid-point in larval development. In ongoing studies, we are investigating whether there is a critical period for rescue of the tap response. However, as mentioned, the isolate effect on behavior is very fragile and is easily disrupted by extraneous stimulation in the incubator at any time during the $4 \mathrm{~d}$ period, and we are hypothesizing that there will not be a critical period for the rescue of the behavior.) To deliver the same number of stimulations to all worms at exactly the same age, a cardboard box containing plates of L3 isolate and colony worms was removed from the incubator, and 30 mechanosensory stimuli were delivered to all plates simultaneously by dropping the box onto a table from a height of $5 \mathrm{~cm} 30$ times at a $10 \mathrm{~s}$ interstimulus interval (ISI). After this stimulation, the box containing the stimulated isolate-raised and stimulated colony-raised worms was returned to the incubator until testing at $4 \mathrm{~d}$ of age.

\section{Scoring and analysis}

Responses to tap were videotaped and later scored using stop-frame video analysis. For each worm videotaped, a tracing was made of the length and width of the worm. Worm length was calculated by measuring the worm from the tip of the head to the tip of the tail. Worm width was calculated by measuring the width of the worm at the midpoint of its body length. A "reversal" was defined as a worm swimming backward in response to the tap within $1 \mathrm{~s}$ after the tap.

Reversals were traced onto acetate sheets and later scanned (ScanJet 3c; Hewlett-Packard, Palo Alto, CA) into a Macintosh (Performa 6200CD; Apple Computers, Cupertino, CA) computer using DeskScan II software. Tracings were measured using NIH Image software, and all data analyses were performed in Statview 4.5 (Abacus Concepts, Berkeley, $\mathrm{CA}$ ). Because differences were found in the length of worms raised under different conditions, all of the response to tap data were reported standardized to a percentage of worm length by dividing the distance each worm reversed by that worm's length and multiplying by 100 .

For spontaneous reversals, 52 colony and 44 isolate worms were each placed alone on agar-filled Petri plates and filmed for 6 min. Backward movements of the body during the 6 min period were counted as spontaneous reversals and scored in the same manner as reversals.

Because the magnitude of the response to tap is affected by a number of factors including temperature, humidity, and age of the agar plates used, a separate N2 group was run on the same day and under the same conditions as the experimental groups for every experiment. Across experiments, there was some variability in the raw scores of the N2 worms as a result of changes in environmental conditions, but the effect of isolation on the response was consistent (isolates significantly smaller than colony). Because we found that the colony/isolate effect was easily disrupted (if the worms experienced even very low levels of vibration during development the effect was lost), we depended on the N2 worms as an indicator of this disruption. If the N2 worms did not show the colony/isolate effect (resulting from a variety of factors including the incubator being bumped, the plates being contaminated, or the worms being sick), the data from that experiment were thrown out, and the experiment was rerun.

When doing statistical analysis on mutant strains of worms (see Figs. $1 a, 3 a-d)$, comparisons were only made within a strain to directly test our experimental manipulation (i.e., colony vs isolate) and not between 


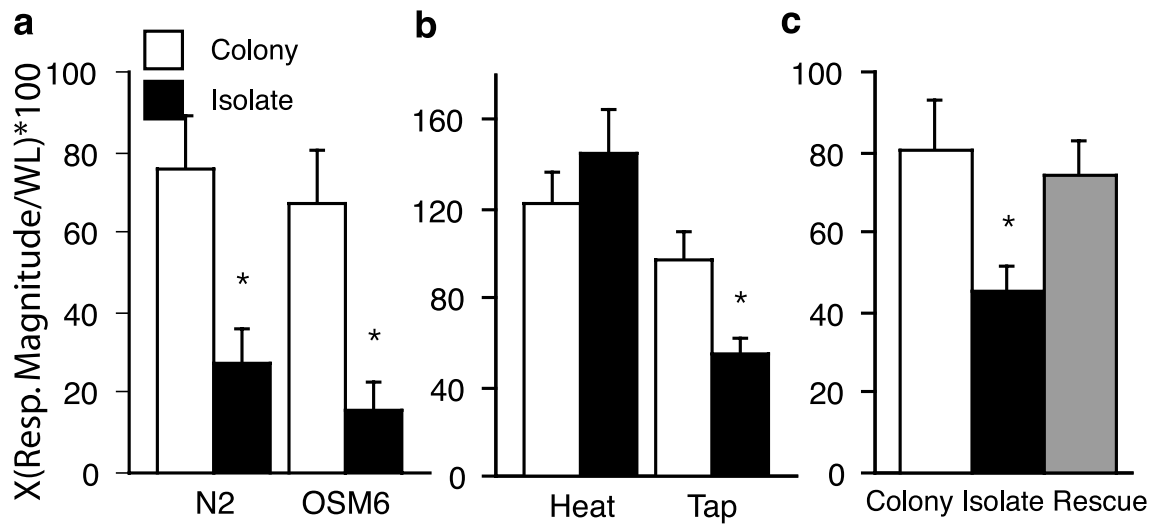

Figure 1. Effects of isolation on tap withdrawal response. $\boldsymbol{a}$, Mean tap response magnitude (Resp. Magnitude) for wild-type (N2) worms and a chemosensory mutant (osm-6). The isolate-raised worms of both strains showed significantly smaller responses to tap than did the colony-raised worms ( $n=20$ per group). $\boldsymbol{b}$, Effects of isolation on reversals to heat. Mean reversal magnitude to tap and to heat probe for isolate-raised and colony-raised worms ( $n=20$ per group). Isolate-raised worms had significantly smaller responses to tap than colony worms. There were no differences between the isolate-raised and colony-raised worms' responses to the heat probe. $c$, Effects of stimulation on isolated worms. Mean tap response magnitude for colony-raised, isolate-raised, L3 stimulated colony-raised worms, and L3 stimulated isolate-raised worms (L3 worms were raised in colony or isolation but were given brief stimulation during the third larval stage). Brief mechanosensory stimulation in $\mathrm{L} 3$ reversed the effects of isolation on behavior. Stimulated isolate-raised responses to tap were larger than the isolate-raised worms and not different from colony-raised or stimulated-colony raised worms. The asterisks represent statistically significant pairs. Error bars represent SEM. WL, Worm length.

strains. We did not make statistical comparisons between strains, because mutations are made on a large number of different genetic backgrounds and may differ from our wild-type worms in a number of ways. Therefore, differences between strains in baseline measures were not relevant for this paper.

\section{Heat probe experiment}

A heated probe (a scalpel blade heated in an alcohol flame until it glowed red) was moved manually into the path of a worm during forward locomotion so that the probe was perpendicular to the body axis of the worm and contacted neither the worm nor the agar surface. The reversal response to the probe was filmed and scored in the same way as the reversals to tap.

\section{Egg counting experiment}

Colony and isolate reared plates were set up as described above. Plates were taken from the incubator at either 66 or $70 \mathrm{~h}$ of age, and the number of worms (for colony plates) and the number of eggs per plate were counted and recorded using a manual counter.

\section{Confocal GFP imaging}

Worms were mounted onto $14 \times 14 \mathrm{~mm}$ three-square slides (one worm per slide; Erie Scientific, Erie, PA) using $12 \mu \mathrm{l}$ of 2,3-butanedione monoxime for paralysis mixed with Sephadex beads (G-50 medium) to prevent worms from being crushed on the microscope stage during imaging. Images were obtained using a Nikon (Tokyo, Japan) Optiphot-2 microscope with an MRC 600 confocal system (Bio-Rad, Hercules, CA) equipped with a krypton/argon laser. GFP was excited using a $488 \mathrm{~nm}$ wavelength laser setting with the emitted light collected by passing through a $\sim 510-550 \mathrm{~nm}$ bandpass filter. The images collected from the MRC 600 were captured in a $768 \times 512$ pixel field of view with the optical sections collected at $0.5 \mu \mathrm{m}$ intervals. Worms were imaged using a $60 \times$ magnification oil lens.

GLR-1::GFP was expressed along the ventral cord. Therefore, images were collected along the posterior portion of the ventral cord from the tail to the vulva. Images of GLR-1::GFP expression were composed of $10-15$ optical sections for each ventral nerve cord segment. Each resultant stack of images was then compiled into a single projection image. These projection images were used for the analyses. The GFP expression in the ventral nerve cord is uniform in thickness. Therefore, the length of GFP expression was measured (Rose et al., 2003).
GFP expressed in the ${ }_{p} m e c-7:: \mathrm{SNB}-1:: \mathrm{GFP}$ strain was captured in a single image stack each composed of $\sim 12-18$ optical sections. Because the GFP in these worms was quite faint, the microscope was set to maximal sensitivity for all worms, and intensity was not measured. Worms with no detectable GFP were discarded $(\sim 13.5 \%$ of the worms distributed evenly across groups). Because the SNB-1::GFP appeared as one to three clustered bright spots, area measurements were used to quantify GFP expression (Rose et al., 2003).

\section{Analysis of confocal images}

GLR-1::GFP. Collected projection images were coded and viewed in NIH Image 1.61. A researcher blind to the condition of the worm measured the number and length of GFP expression in the image. Measurements were entered in Statview 4.5 for statistical analysis.

${ }_{p}$ mec-7::SNB-1::GFP. Projection files were coded before analysis so that all measurements were made by a researcher blind to the condition of the worm. Single projection images were opened in NIH Image (as above) and viewed as a binary image with the threshold adjusted to allow for measuring of faint images. Area measurements for each region of GFP expression were calculated by outlining the edge of the region and using the area measure function. Area measurements were entered into Statview 4.5 for statistical analysis.

Final figures for GFP images were generated using Adobe Photoshop 7.0 (Adobe Systems, San Jose, CA).

\section{Statistical analysis}

In experiments in which only two groups were run, unpaired $t$ tests were used to determine significance. If multiple groups were run, an ANOVA with Fisher's planned least significant difference (PLSD) planned comparisons were used. For all experiments, $\alpha$ was set to $p \leq 0.05$.

\section{Results}

\section{Isolation decreases mechanosensory responses}

In our initial experiment, we compared the response to tap in 4 -d-old adult worms that had been raised in isolation $(n=19)$ to that of worms raised in groups or colonies $(n=20)$. Worms that developed under isolated conditions responded to tap with significantly smaller responses than worms that developed in the presence of other worms (isolate, $19.63 \pm 11.65$; colony, $73.28 \pm$ $\left.5.54 ; t_{(37)}=4.088 ; p \leq 0.0002\right)$. A number of mutant strains tested showed the same results as wild-type worms; these included cat-2, nmr-1, npr-1, avr-14, and daf-2 (see supplemental material, available at www.jneurosci.org).

C. elegans receive sensory input through mechanosensory, chemosensory, and thermosensory systems. Worms that are isolated throughout development experience an environment of impoverished sensory stimulation, deprived of both mechanosensory and chemosensory stimulation from encounters with other worms. We hypothesized that the effect of isolation on the initial response to tap was caused by decreased mechanosensory input during development. To rule out the possibility that a lack of chemosensory stimulation plays a role in the decreased response to tap in isolated worms, in a second experiment, the responses of a mutant strain of worms osm-6 (p811)V, deficient in chemosensation, were compared with the responses of a new group of wild-type (N2) worms. The magnitudes of the standardized reversal response to tap for the colony and isolate groups of $\mathrm{N} 2$ and osm- 6 worms are shown in Figure $1 a$. An overall ANOVA 
comparing mean tap response magnitude for each group showed a significant difference between rearing conditions $\left(F_{(3,76)}=\right.$ $6.861 ; p \leq 0.0004)$. Fisher's planned comparisons indicated significant differences in response magnitudes between isolate- and colony-raised worms in both the N2 strain $(p \leq 0.0034)$ and the osm-6 strain ( $p \leq 0.0017$ ). Based on these results, it is highly unlikely that a lack of chemosensory stimulation plays a role in the decreased response to tap of isolated worms. An additional experiment with "worm-conditioned" plates, which held colonies of worms for $4 \mathrm{~d}$ before they were removed and the plate then used for rearing isolates (conditioned plate colony response to tap, $X=246.73 \pm 44.0$; conditioned plate isolate response to tap, $\left.X=133.28 \pm 23.58 ; t_{(34)}=2.27 ; p=0.029\right)$, confirmed that a lack of chemosensory cues from conspecifics were not responsible for the alteration in response to tap.

It is possible that isolation had effects on general sensory or motor abilities and was not specific to the mechanosensory system. To test this, we looked at other behaviors that use the same neural pattern generator (interneurons, motor neurons, and muscles) but different sensory inputs to test this hypothesis. When worms are confronted with a heated probe, they swim backward, away from the probe (Wicks and Rankin, 1997). The magnitudes of the standardized reversal response to tap and to heat probe for the colony and isolate groups were compared using an ANOVA, which resulted in a significant difference between rearing condition, $\left(F_{(3,76)}=7.140 ; p \leq 0.0003\right)$ (Fig. 1b). Fisher's planned comparisons showed that isolate-raised worms gave significantly smaller responses to tap than did colony-raised worms ( $p \leq 0.047)$, but there were no significant differences in the response magnitudes to the heat probe between colony and isolate-raised worms ( $p \leq 0.2745)$.

When worms are placed on an agar plate without food, they swim over the surface of the agar foraging for food. As part of their normal movement, worms frequently stop swimming forwards and swim backward for a short period of time before resuming forward locomotion. This period of swimming backward has been called a spontaneous reversal (Chiba and Rankin, 1990). The tap sensory neurons do not play a role in the frequency or magnitude of these reversals (Wicks and Rankin, 1995, 1997). When spontaneous reversals were examined in colony- $(n=52)$ and isolate- $(n=44)$ raised worms, there were no significant differences in either the frequency of spontaneous reversals (colony-reared, $3.37 \pm 2.55$; isolate-reared, $2.98 \pm 1.7 ; t_{(93)}=$ $-0.49 ; p \leq 0.62$ ) or the magnitude of spontaneous reversals (colony-reared, $315.24 \pm 256.5$; isolate-reared, $338.24 \pm 184.3$; $\left.t_{(93)}=0.87 ; p \leq 0.39\right)$.

Together, these results indicate that neither the thermosensory system nor spontaneous reversal circuits were affected by isolation and that the basic functioning of the command interneurons and the motor neurons was not altered in worms raised in isolation. Thus, we hypothesize that the most likely locus for the behavioral effects of isolation is the tap sensory neurons themselves and/or the presynaptic or postsynaptic elements of their synapses onto the interneurons.

\section{Mechanical stimulation can reverse the effects of isolation on behavior}

The experiments described above suggested the hypothesis that the lack of mechanical stimulation from contacts between conspecifics led to a decreased responsiveness to mechanical stimulation. To test this hypothesis, we tried to reverse the effects of isolation with stimulation of isolated worms during the midpoint of larval development. There were four groups of worms in this experiment, normal colony-raised $(n=42)$ and isolate-raised groups $(n=36)$, as well as stimulated isolate-raised $(n=39)$ and stimulated colony-raised $(n=40)$ groups that were given 30 mechanosensory stimuli at a $10 \mathrm{~s}$ interstimulus interval during the third larval stage of development (L3). There were significant differences in the initial response to tap between these colonyraised, isolate-raised stimulated, isolate-raised, and stimulated colony-raised worms $\left(F_{(3,153)}=4.6 ; p=0.004\right)$ (Fig. 1c). Fisher's PLSD tests showed that colony-raised, stimulated colony-raised, and stimulated isolate-raised worms had responses significantly larger than those of isolate-raised worms ( $p=0.001, p=0.002$, and $p=0.05$, respectively). Thus, these experiments show that mechanical stimulation during larval development could reverse the behavioral effects of isolation on the response to a single tap. [Anecdotally, we observed that high levels of activity in and around the incubator at any time during these experiments would eliminate the differences between the colony- and isolateraised worms. In pilot experiments, raising worms in isolation with Sephedex beads rescues the effect of isolation on behavior (data not shown).]

\section{Isolation slows development and decreases body size}

Over the course of the initial behavioral experiments, the isolateraised worms appeared noticeably smaller than the colony-raised worms. To document this, measurements of body size were analyzed. Worm length was measured from the tip of the head to the tip of the tail using video images. Worm width was measured at the midpoint of each worm's body length. An unpaired $t$ test showed that isolate-raised worms were significantly shorter than colony-raised worms $\left(t_{(478)}=3.866 ; p \leq 0.0001\right)$ (Fig. $\left.2 a\right)$ and that isolate-raised worms were significantly narrower than colony-raised worms $\left(t_{(438)}=5.100 ; p \leq 0.0001\right)$ (Fig. $\left.2 b\right)$. This effect of isolation on size was confirmed in osm- 6 worms. Isolateraised osm-6 worms $(X=320.57 \pm 3.58)$ were significantly shorter than colony-raised worms $\left(X=349.67 \pm 3.31 ; t_{(49)}=\right.$ 5.97; $p<0.0001)$ and in colony and isolate worms raised on plates conditioned for $4 \mathrm{~d}$ with colonies of worms (conditioned colony worm length, $X=293.78 \pm 3.13$; conditioned isolate worm length, $\left.X=264.46 \pm 8.05 ; t_{(34)}=3.39 ; p=0.002\right)$. The data from chemosensory mutants together with the observation that isolate worms raised on chemically conditioned plates were still smaller than colony-reared worms (conditioned isolate, $X=$ $264.46 \pm 34.17$; conditioned colony, $X=293.78 \pm 13.28$; $t_{(34)}=$ 3.39; $p=0.002$ ) suggests that a deficit in mechanosensory input alone (without a deficit in chemosensory input) is sufficient to lead to smaller worms. A number of other mutant strains tested showed the same results as wild-type worms, isolates were smaller than colony worms. These included cat-2, nmr-1, npr-1, avr-14, and daf-2 (see supplemental material, available at www.jneurosci.org).

We hypothesized that the size differences between colonyand isolate-raised worms might be the result of different rates of development in the two conditions. To measure rate of development, we recorded when egg-laying began. At $20^{\circ} \mathrm{C}$, worms normally begin to lay eggs at $\sim 65 \mathrm{~h}$ of age (Byerly et al., 1976). To determine whether isolation altered the rate of development, the number of eggs laid per worm was compared between colonyraised and isolate-raised worms at 66 and $70 \mathrm{~h}$ of age. Neither of the two groups laid many eggs at $66 \mathrm{~h}$ of age; however, an ANOVA indicated that worms that developed under isolated conditions had significantly fewer eggs per worm than colony-raised worms at $70 \mathrm{~h}$ of age $\left(F_{(1,42)}=33.61 ; p \leq 0.0001\right)$ (Fig. $\left.2 c\right)$. Thus, isolateraised worms are either somehow defective in egg laying behavior or they are slower in their rate of development. 
a

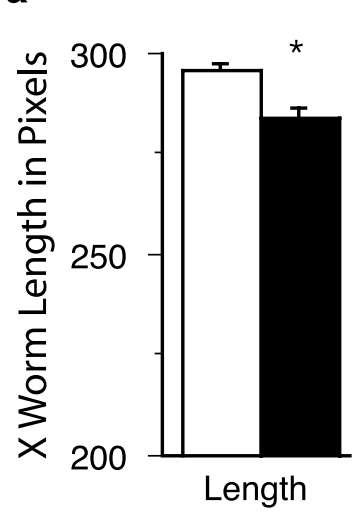

b

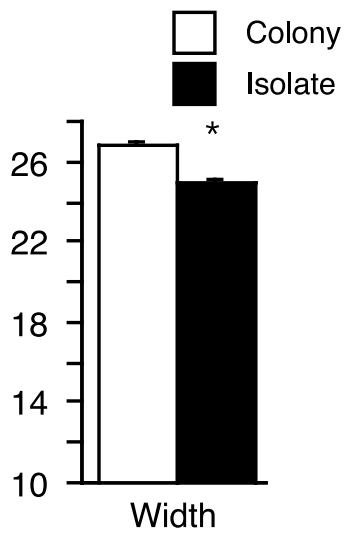

d

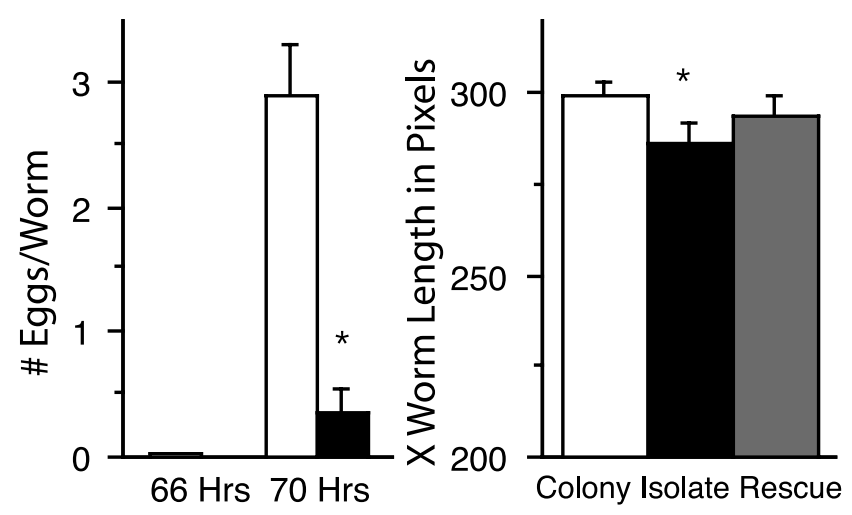

Figure 2. Effects of isolation on development. isolate-raised worms were significantly shorter $(\boldsymbol{a})$ and narrower $(\boldsymbol{b})$ than worms raised in colonies ( $n=240$ per group). $\boldsymbol{c}$, Mean number of eggs per worm for isolate ( $n=22$ worms) and colony-reared worms ( $n=22$ colonies for total of $1480 \mathrm{~N} 2$ worms) at 66 and $70 \mathrm{~h}$ of development. At $70 \mathrm{~h}$, there were significantly more eggs per worm for the colony-reared worms than for the isolate-reared worms. $\boldsymbol{d}$, Brief mechanosensory stimulation during $L 3$ did not reverse the effects of isolation on development. Isolate worms were significantly shorter than colony worms and stimulated colony worms, worms raised in isolation but stimulated in L3 were significantly different from the colony-raised worms, and stimulated colony-raised worms but not significantly different from the isolate-raised worms. The asterisks represent statistically significant pairs. Error bars represent SEM.

To determine whether the effects of isolation on development could be reversed by stimulation during larval development, the lengths of the isolate-raised, colony-raised, stimulated colonyraised, and stimulated isolate-raised worms described previously were compared. An ANOVA showed overall differences in length among the four groups $\left(F_{(3,153)}=5.87 ; p=0.0008\right)$ (Fig. $2 d$ ). Fisher's PLSD comparisons showed that colony-raised worms were significantly longer than isolate-raised $(p=0.0005)$ and stimulated isolate-raised worms $(p=0.043)$; there were no significant differences between isolate-raised and stimulated isolate-raised worms ( $p=.0 .12)$; stimulated colony-raised worms were not significantly different from colony-raised worms ( $p=0.99)$ but were significantly different from isolate-raised worms ( $p=0.0006)$ and stimulated isolate-raised worms ( $p=$ 0.049 ). In pilot studies, we have delivered as many as 400 mechanical stimuli to isolate-raised worms in L3 and have not rescued the effects of isolation on worm length (data not shown). Thus, unlike the response to tap, brief mechanical stimulation during the third larval stage did not rescue the effects of isolation on development.

Because these experiments suggested that isolate worms were delayed in development compared with colony worms, we wanted to ensure that the effect of isolation on response to tap was not simply a result of this delay we compared the response to tap for colony worms run early in a test session with isolate worms tested several hours later in the test session. We still found that isolate-raised worms $(X=44.52 \pm 11.45)$ showed significantly smaller responses to tap than did colony-raised worms $(X=$ $\left.191.58 \pm 39.33 ; t_{(54)}=3.26 ; p=0.002\right)$. Thus, the effects of isolation on tap are not simply because of a small delay in development of the isolate worms.

GLR-1 is critical for the changes in the response to tap but not changes in size in isolate-reared worms

Previous analyses of the neural circuit underlying the response to tap using laser ablation determined that the circuit is composed of five mechanosensory neurons, one putative stretch receptor, and five pairs of interneurons (Wicks and Rankin, 1995). All reversal behaviors in C. elegans use at least some of the interneurons and the same motor neurons as the response to tap (Wicks and Rankin, 1997). The data showing that isolation does not affect spontaneous reversals or reversals to the heat probe suggest that the ability of the interneurons and motor neurons to produce the reversal behavior was not altered by isolation. Thus, the most likely sites altered by isolation are the presynaptic or postsynaptic components of the synapses between the mechanosensory neurons and the interneurons.

There is accumulating evidence that the chemical synapses from the mechanosensory neurons of the tap withdrawal circuit onto the interneurons are glutamatergic. Several laboratories have isolated genes that code for glutamate receptors. These include glr-1 [homologous to AMPA type (Hart et al., 1995; Maricq et al., 1995)], avr-15 [glutamate-gated Cl channel (Dent et al., 1997)], and nmr-1 [homologous to NMDA-type channels (Brockie et al., 2001)]. All three of these glutamate receptor types are expressed in one or more of the four command interneurons (AVA, AVB, AVD, and PVC) that play a central role in the tap withdrawal response. Other evidence for glutamatergic transmission comes from investigations of eat-4, a gene for a vesicular glutamate transporter (homologous to mammalian vesicular glutamate transporter-1) that is expressed in several pharyngeal neurons and in the sensory neurons that transduce tap (Avery, 1993; Lee et al., 1999; Bellocchio et al., 2000, Rose et al., 2003). Thus, there is sufficient evidence to support the hypothesis that the chemical synapses between the touch cells and the interneurons are glutamatergic.

In our studies of long-term memory for habituation in adult worms, we found that the $g l r-1$ glutamate receptor was critical for long-term memory for habituation training (Rose and Rankin, 2003). Worms with mutations in $g l r-1$ did show normal shortterm habituation but did not show $24 \mathrm{~h}$ retention of habituation training. Because of this finding, we hypothesized that $g l r-1$ would be important of the effects of isolation on the response to tap. To test whether $g l r-1$ plays a role in the decreased response to tap in isolated worms, the responses of a putative null strain $g l r-1(n 2461)$ as well as a $g l r-1$ rescue strain ( $k p 537)$ were compared with the responses of wild-type worms. The magnitudes of the standardized reversal response to tap for the colony and isolate groups of $\mathrm{N} 2, g l r-1$, and $g l r-1$ rescue worms are shown in Figure $3 a$. An overall ANOVA comparing mean tap response magnitude for each group showed a significant difference between rearing conditions $\left(F_{(5,476)}=5.01 ; p=0.0002\right)$. Fisher's planned comparisons indicated significant differences in response magnitudes between isolate- and colony-raised worms in 
a

\section{b}
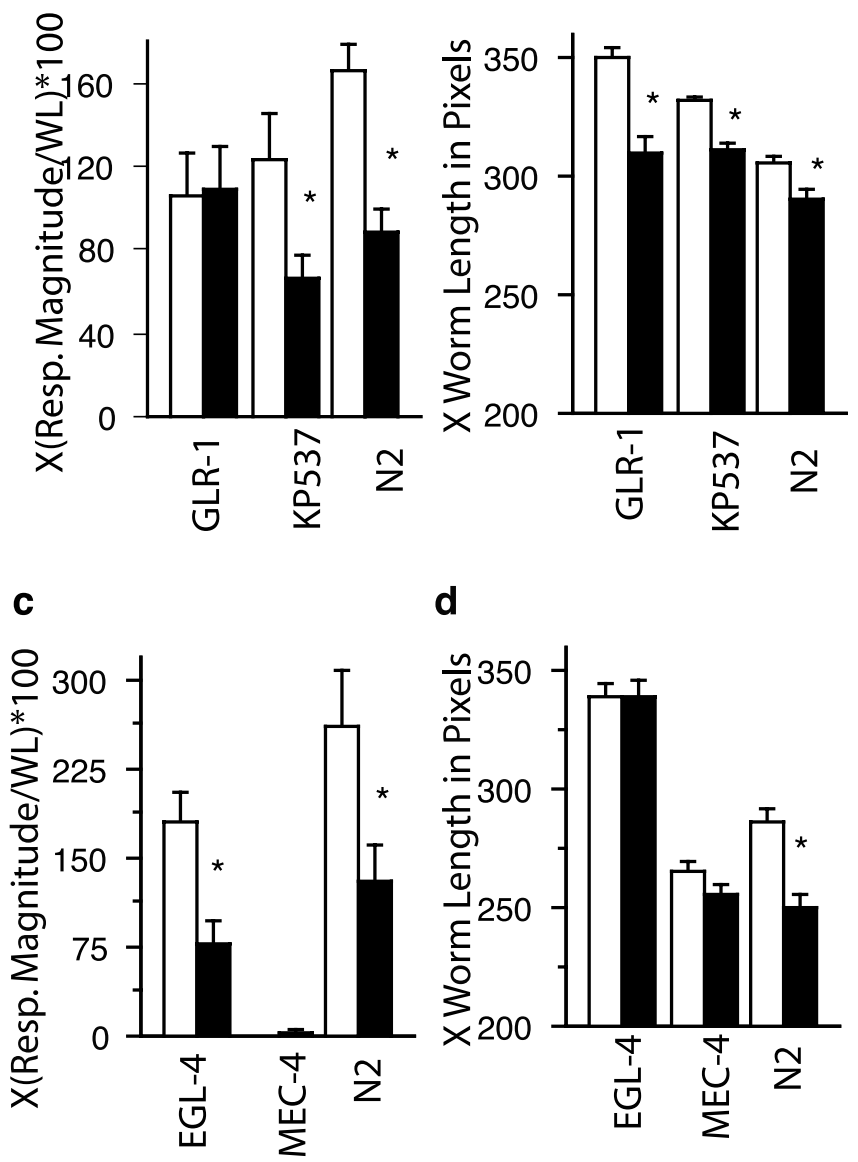

Figure 3. Genetic analyses of effects of isolation. $\boldsymbol{a}$, Role of $\mathrm{g} / \mathrm{r}-1$ in the effects of isolation on initial response to tap. Isolate raised N2 $(n=95)$ and kp537 ( $g / r-1$ rescue; $n=81$ ) worms showed significantly smaller responses to tap than N2 $(n=106)$ and $k p 537$ ( $g$ lr- 1 rescue; $n=$ 81) worms raised in colonies. For the glr-1 worms, there were no significant differences in the isolate $(n=57)$ and colony $(n=67)$ worms response to tap. $\boldsymbol{b}$, Role of $\mathrm{glr}-1$ in the effects of isolation on worm length. Isolate raised N2, g/r-1 rescue (kp537), and g/r-1 groups were all significantly shorter than colony raised conspecifics. c, Role of mec- 4 and egl- 4 in the effects of isolation on initial response to tap. Isolate raised N2 $(n=13)$ and egl-4 $(n=20)$ worms showed significantly smaller responses to tap than $\mathrm{N} 2(n=13)$ and egl-4 $(n=22)$ worms raised in colonies. The mec -4 worms did not respond to tap and so there were no significant differences in the isolate ( $n=19)$ and colony ( $n=18)$ worms response to tap. $\boldsymbol{d}$, Role of mec -4 and egl-4 in the effects of isolation on worm length. Isolate raised N2 worms were significantly shorter than colony raised conspecifics. In contrast, there were no significant differences in worms length when mec-4 and egl-4 worms raised in isolation were compared with mec-4 and egl-4 worms raised in colonies. The asterisks represent statistically significant pairs. Error bars represent SEM. Resp. Magnitude, Response magnitude; WL, worm length.

the N2 strain $(p=0.0002)$ and the $g l r-1$ rescue strain $(p=0.015)$ and no differences between isolate- and colony-raised worms in the $g l r-1$ strain $(p=0.88)$. Based on these results, it appears as though the decreased response to tap seen in isolated worms is mediated by the $g l r-1$ type glutamate receptors.

Because N2 worms reared in isolation were significantly smaller than $\mathrm{N} 2$ worms reared in colonies, we measured the worm length and width for the $\mathrm{N} 2$, the $g l r-1$, and the $g l r-1$ rescue strain of worms and found that all three strains that were raised in isolation were significantly shorter than the same age worms raised in colonies $\left(F_{(5,476)}=31.9 ; p<0.0001 ; \mathrm{N} 2, p=0.01 ; g l r-1\right.$, $p=0.0001 ; g l r-1$ rescue, $p=0.001)$ (Fig. $3 b)$. Thus, although $g l r-1$ is critical for the effects of isolation on the response to tap, it is not critical for the effect of isolation on size.
Effects of isolation on worm length are mediated by egl-4

The finding that GLR-1 worms showed the effects of isolation on the initial response to tap but not on worm length might mean that the touch cells are not important for the effects of isolation on worm length, or it might mean touch receptors use a nonGLR-1 pathway to affect size. To test the hypothesis that the touch cells are critical for the decreased worm length in isolates, we examined the effects of isolation on the response to tap (there should be no response to tap in either condition) and on size in mec-4 worms. MEC-4 is a gene that codes for an amiloridesensitive sodium channel that is expressed in the six touch sensory neurons; mec-4 worms are touch insensitive (Driscoll and Chalfie, 1991). If the effects of isolation on worm size are caused by lack of activation of the touch cells, then mec- 4 worms should show no effects of isolation on either measure.

In a paper investigating the effects of chemosensory deprivation on C. elegans, Fujiwara et al. (2002) showed that genetic abnormalities in C. elegans chemosensory organs that decreased sensory input led to decreased body size. This decrease in body size was mediated by EGL-4, a gene that encodes a cGMPdependant protein kinase that acts via the TGF- $\beta$ signaling pathway. EGL-4 is expressed in a number of neurons in the head, in some muscle cells, and in the intestine (Hirose et al., 2003). Although Fujiwara et al. (2002) showed that EGL-4 is activated by sensory input to regulate size, it is not clear how it does this. We hypothesized that egl-4 might also be responsible for the decreased size seen in worms raised in isolation. If this hypothesis is correct, then egl-4 worms should still show the effect of isolation on the initial response to tap but not on size.

To test these hypotheses, we raised N2, mec-4, and egl-4 worms in isolation and in colonies. At $4 \mathrm{~d}$ of age, we compared initial response to tap and worm length. An overall ANOVA comparing mean tap response magnitude for each group showed a significant difference between rearing conditions $\left(F_{(5,99)}=19.2\right.$; $p<0.0001$ ) (Fig. 3c). Fisher's planned comparisons indicated significant differences in response magnitudes between isolateand colony-raised worms in the $\mathrm{N} 2$ strain $(p=0.0006)$ and in the egl-4 strain ( $p=0.0007)$ and no differences between isolate- and colony-raised worms in the mec-4 strain $(p=0.93)$. An overall ANOVA comparing mean worm length for each group also showed a significant difference between rearing conditions $\left(F_{(5,99)}=53.5 ; p<0.0001\right)$ (Fig. 3d). Fisher's planned comparisons indicated significant differences in worm length between isolate- and colony-raised worms in the N2 strain $(p=0.0003)$ and no differences between isolate- and colony-raised worms in the egl-4 strain $(p=0.96)$ or in the mec- 4 strain $(p=0.22)$. Thus, $\mathrm{N} 2$ worms show effects of isolation on both response to tap and worm length, egl-4 worms show effects of isolation on response to tap and not on worm size, whereas mec- 4 worms that do not respond to tap also do not show effects of isolation on worm size.

\section{Isolation alters both presynaptic and postsynaptic gene expression}

Because $g l r-1$ receptors are critical for the decreased responses to tap seen in isolate-reared worms, we investigated whether the amount of stimulation during development would alter the expression of $g l r-1$. To do this, we raised a strain of worms expressing chimeric receptors made up of GLR-1 tagged with the green fluorescent protein (GLR-1::GFP), which has been used to visualize synapses in C. elegans (Rongo and Kaplan, 1999), under colony and isolate conditions. We focused on synapses in the posterior ventral cord because these are the easiest to visualize. From projected confocal images (each image represented a por- 
a
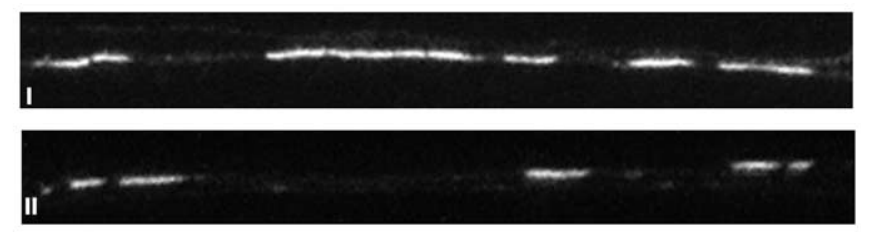

b

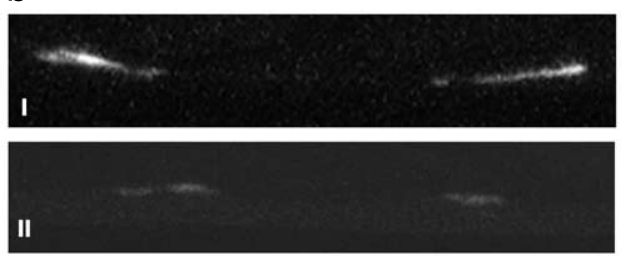

Figure 4. Changes in glutamate receptor and vesicle concentration. $\boldsymbol{a}$, Effects of isolation on GLR-1::GFP expression in the ventral nerve cord. Representative confocal image of a segment of the ventral nerve of a colony-raised worm (i) and an isolate-raised worm (ii) expressing GLR-1::GFP. White areas are clusters of GLR-1::GFP expressed in the ventral nerve cord. These are probably on or in the processes of interneurons of the tap withdrawal response (26). Isolateraised worms showed less total GLR-1::GFP expression per image than did colony-raised worms. $\boldsymbol{b}$, Effects of isolation on ${ }_{p}$ mec-7::SNB-1::GFP expression in the ventral nerve cord. A representative confocal image of a segment of the ventral nerve cord of a colony-raised worm (i) and an isolate-raised worm (ii) expressing ${ }_{p}$ mec-7::SNB-1::GFP is shown. White areas are GFP expressed in the sensory terminals of the tail sensory neurons (PLML and PLMR; 27). Isolate-raised worms showed significantly less ${ }_{p}$ mec-7::SNB-1::GFP expression in the ventral cord than did colony-raised worms.

tion of the posterior ventral cord with two to four images per worm; $n=9$ per group), we compared the total amount of GLR-1::GFP expression for each image. There was no significant difference in the number of GLR-1::GFP clusters (isolates, $19.39 \pm 8.1$; colony, $\left.18.86 \pm 8.6 ; t_{(58)}=0.24 ; p \leq 0.8\right)$; however, isolate-reared worms expressed significantly smaller GLR-1::GFP average length per image than colony-reared worms (isolates, $78.33 \pm 6.63$; colony, $\left.138.45 \pm 10.53 ; t_{(58)}=4.9 ; p \leq 0.0001\right)$ (Fig. 4a). The data on total GLR-1::GFP per image suggest that fewer $g l r-1$ receptors were expressed on the interneurons of isolate-raised worms than on the interneurons of colony-raised worms.

Because we found a difference in the expression of $g l r-1$ receptors as a result of rearing, we investigated whether rearing condition would alter the presynaptic terminals of the tap withdrawal response. Therefore, in a second strain of worms, we looked at GFP expression of the gene for C. elegans snb-1, a protein associated with synaptic vesicles that plays a role in regulating vesicle fusion at the synaptic terminal. SNB-1::GFP was expressed under the control of the mec-7 promoter that produces expression in the six mechanosensory neurons of the tap withdrawal circuit (Nonet, 1999). This construct, ${ }_{\mathrm{p}} m e c-7:: \mathrm{SNB}-1:: \mathrm{GFP}$, allowed us to use confocal imaging to visualize and compare the tap sensory neuron terminals in the ventral nerve cords of isolate-raised $(n=23)$ and colony-raised worms $(n=24)$. The fluorescence appeared as one or two large patches just posterior to the vulva. We measured the total area of the patches and found that isolate-raised worms had significantly smaller areas of fluorescence than did colony-raised worms (isolated, $40.35 \pm 6.6$; colony, $68.21 \pm 8.1 ; t_{(45)}=2.64 ; p \leq 0.01$ ) (Fig. $4 b$ ). These results suggest that the amount of synaptobrevin and, by implication, the number of synaptic vesicles in the terminals of these mechanosensory neurons was determined by the amount of activity in this circuit across development.

The effects of isolation on rate of development raised the pos- sibility that the differences between colony- and isolate-raised worms seen in the amount of GLR-1::GFP fluorescence expressed in the posterior ventral cord were the result of a developmental difference between the two groups. In pilot studies, we determined that 74-h-old isolate-raised worms lay approximately the same number of eggs per worm as 70-h-old colony worms. Imaging of GLR-1::GFP worms took place over the course of $1 \mathrm{~d}$, and so the results from the first half of the day were compared with the second half of the day to determine whether expression in isolate worms changed when worms were $3-5 \mathrm{~h}$ older. There were no significant differences in amount of GFP expression across the day (morning, $102.22 \pm 9.5$; afternoon, $112.95 \pm 12.48$; $t_{(52)}=$ $0.67 ; p=0.5)$. Therefore, the differences we observed in GLR-1::GFP expression between colony and isolate worms were not simply a side effect of slower development of the colony worms.

\section{Brief stimulation during $\mathrm{L} 3$ reverses the postsynaptic effect of isolation}

To determine whether brief stimulation during larval development would rescue the decreased expression of $g l r-1$ or of synaptobrevin in isolated worms, stimulated isolate-raised worms were given the same brief mechanical stimulation in L3 as described previously and compared with isolate-raised and colony-raised worms. There was a significant difference between the three groups in GLR-1::GFP expression $\left(F_{(2,62)}=3.82 ; p=0.03\right.$ ) (Fig. $5 a)$. Fisher's PLSD tests showed isolate-raised worms had significantly less GFP expression than both colony-raised $(p=0.01)$ and stimulated isolate-raised ( $p=0.02$ ) worms (pilot experiments showed that stimulating colony worms had no effect of GLR-1::GFP expression; data not shown). When the amount of SNB-1::GFP expressed was examined, there was also a significant difference between the three groups $\left(F_{(2,61)}=4.37 ; p=.017\right)$ (Fig. 5b). However, no apparent rescue effect was seen. Fisher's PLSD tests showed that both isolate-raised and stimulated isolate-raised had significantly less SNB-1::GFP than colonyraised worms ( $p=0.01, p=0.02$, respectively). There was no significant difference in the area of SNB-1::GFP expression between isolate-raised and stimulated isolate-raised worms. Thus, brief stimulation during L3 that rescues the behavioral effects of isolation greatly increases the level of $g l r-1$ expression on the interneurons of isolated worms and does not significantly alter the effects of isolation on synaptobrevin expression in the sensory neurons.

These results also indicated that the stimulation that the worms received in $\mathrm{L} 3$ did not completely reverse the effects of isolation on the synaptic elements and that there might still be deficits in mechanosensory behavior as a result of the apparent low level of vesicles in isolates. We hypothesized that the stimulated isolate worms might show deficits in response to repeated stimulation (habituation). We repeated the L3 stimulated isolate experiment described above, only instead of testing worms with a single tap at $4 \mathrm{~d}$ of age, we gave worms 30 tap stimuli at a $10 \mathrm{~s}$ interstimulus interval. As expected, we found that there were significant differences in the initial response to $\operatorname{tap}\left(F_{(2,99)}=8.45\right.$; $p=0.0004)$. We found that although the responses from stimulated isolate worms differed from isolate worm responses ( $p=$ $0.007)$, they did not differ from colony worms $(p=0.185)$ on the initial response to tap (colony vs isolate; $p=0.0001$ ). There was a different story for habituation: when we examined the overall average response sizes across the 30 stimuli, we again found significant differences $\left(F_{(2,99)}=5.93 ; p=0.0004\right)$. However, the pattern was different. Stimulated isolate worms had significantly 
a

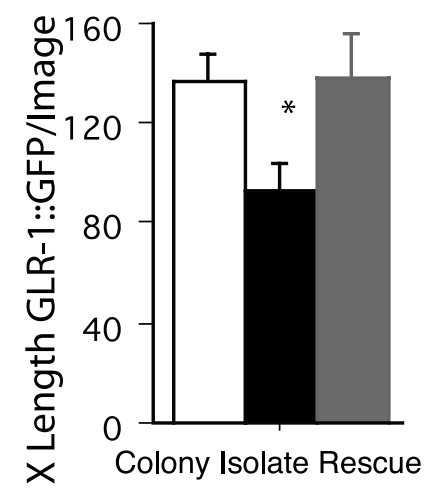

b

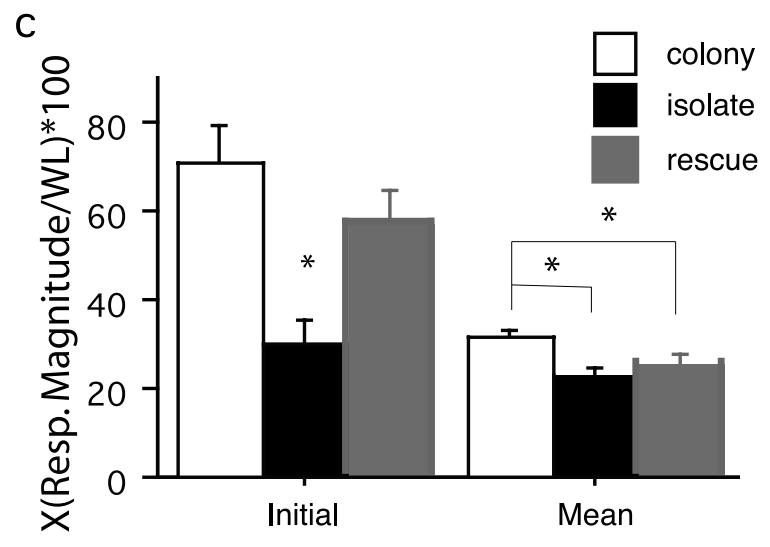

Figure 5. Effects of brief stimulation at $L 3$ on presynaptic and postsynaptic terminals. $\boldsymbol{a}$, Mean length of GLR-1::GFP expression per image in the ventral nerve cord for colony-raised $(n=26)$, isolate-raised $(n=20)$, and L3 stimulated isolate-raised ( $n=19)$ worms. Isolateraised worms showed significantly less GFP expression than either stimulated isolate-raised or colony-raised worm. $\boldsymbol{b}$, Mean area of ${ }_{p}$ mec-7::SNB-1::GFP expression in the ventral nerve cord for colony-raised $(n=20)$, isolate-raised ( $n=17)$, and L3 stimulated isolate-raised $(n=11)$ worms. Both isolate-raised worms and stimulated isolate-raised worms showed significantly less SNB-1::GFP expression than colony-raised worms. c, When colony-raised (white bars; $n=$ 31 ), isolate-raised (black bars; $n=36$ ), and L3 stimulated isolate-raised (gray bars; $n=35$ ) worms were given 30 stimuli at a $10 \mathrm{~s}|S|$ the results showed that stimulated isolates were not the same as colony worms. The first set of three bars (initial) shows the initial response \pm SE to tap. Here, the isolate worms gave significantly smaller responses than either colony or stimulated-isolate worms. The second set of bars (mean) represents the mean \pm SE of responses to 30 stimuli delivered at a $10 \mathrm{~s} I \mathrm{SI}$. These data show that colony worms gave significantly larger responses than did either stimulated isolate or isolate worms. There were no differences between the stimulated isolate and the isolate groups. The asterisks represent the group that differs significantly from the other two groups on the figure. Resp. Magnitude, Response magnitude; WL, worm length.

smaller average responses than did colony worms $(p=0.02)$ and had similar average responses to isolate worms ( $p=0.31$; colony vs isolate, $p=0.001$ ) (Fig. $5 c$ ).

\section{Discussion}

When C. elegans are raised in isolation without the stimulation produced by conspecifics, the response to a mechanosensory stimulus (tap) is greatly decreased. However, other behaviors (the reversal response to a heat probe and spontaneous reversals) that use the same interneurons and motor neurons are not altered by isolation, suggesting that this is a response or circuit-specific effect of isolation. We hypothesized that in the absence of conspecific interactions, there was reduced stimulation of the neural circuit underlying the response during development, which led to a weak synapse developing between the mechanosensory neurons and the interneurons. This was supported by the observations that mechanosensory stimulation during L3 reversed the effects of isolation on the response to tap and that there were fewer synaptic vesicles in the sensory neurons and fewer $g l r-1$ receptors on the interneurons.

In addition to having effects on the tap response, isolation led to either a slowing or alteration of development such that isolatereared worms showed a slower onset of egg laying and were smaller than age-matched colony worms. The overall number of eggs laid by isolate-raised and colony-raised worms has not been examined, so it is not clear whether the effect is merely a delay in the onset of egg-laying, or whether there is a reduction in the overall number of eggs. These results are consistent with Fujiwara et al. (2002) who showed genetic abnormalities in C. elegans chemosensory organs that decreased sensory input led to decreased body size. There is also evidence that the amount of sensory input experienced by a worm alters lifespan. Mutations that disrupt sensory cilia and laser ablation of chemosensory cells reduce chemosensory input and lead to a dramatic increase $(>50-100 \%)$ in worm lifespan (Apfield and Kenyon, 1999). The reduction in overall sensory input experienced by worms raised in isolation suggests the hypothesis that isolate-raised worms might live longer than colony-raised worms. It is likely that under normal conditions, many different sensory inputs are integrated to influence body size and possibly lifespan.

The tap sensory neurons are necessary for the effects of isolation on both the initial response to tap and on worm length; regardless of rearing condition, mec-4 worms that are touch insensitive show no response to tap and no difference in worm length. However, the effect of isolation on the response to tap can be dissociated from the effects of isolation on development in two ways. First, the amount of stimulation required to rescue the effect: the initial response to tap was rescued by 30 taps during L3, this same stimulation did not rescue the effects of isolation on size. Second, different genes are critical for the effects of isolation on response to tap and on worm length. For the effects of isolation on the initial response to tap, glutamate transmission from the touch cells onto the command interneurons is necessary; $g l r-1$ worms showed no effect of isolation on the response to tap, and GLR-1::GFP expression was decreased in worms raised in isolation. For the effects of isolation on worm length, the GLR-1 pathway is not important; $g l r-1$ worms showed the effects of isolation on worm length. The effects of isolation on worm length are mediated by egl-4 that is somehow regulated by touch cell activity; neither egl-4 nor mec-4 worms showed effects of isolation on worm length. It is not clear how sensory systems regulate body size; however, both chemosensory input (Fujiwara et al., 2002) and mechanosensory input (shown in these studies) regulate size through an EGL-4 pathway. Together, these data dissociate the effects of isolation on a behavior and on worm size into two separate genetic pathways dependent on touch cell activity: one works via GLR-1 and the other via EGL-4. This leads to the hypothesis that these are independent effects of developing in an environment with impoverished sensory input.

When we examined the synapses between the sensory neurons and interneurons of the tap-withdrawal circuit, we saw that isolation lowered total GFP expression in both. The data suggest that neither the level of $g l r-1$ expression nor the level of snb-1 expression in this circuit are hard wired but rather are determined by the level of stimulation during development. Lack of stimulation during the course of development led to less synaptobrevin (fewer vesicles) in the presynaptic terminals and fewer $g l r-1$ receptors on the postsynaptic membrane. Brief stimulation during 
larval development led to a large increase in the expression of postsynaptic receptors but did not affect presynaptic synaptobrevin, indicating that postsynaptic changes occur before presynaptic changes. Preliminary data from another experiment suggest that higher levels of stimulation than used here distributed over development in isolated worms do lead to increases in synaptobrevin expression. These data suggest that the amount of stimulation received during development does not lead to a change in the number of synapses but rather an alteration of the strength of existing ones.

The low expression of synaptobrevin in isolate and stimulated-isolate worms is thought to reflect a low number of vesicles in the stimulated isolate worms; this lowered synaptobrevin expression was correlated with more rapid habituation than in colony worms. This finding is strikingly similar to observations of worms with a mutation in the glutamate vesicular transporter eat-4 (Rankin and Wicks, 2000). These eat-4 worms presumably had less neurotransmitter in the touch cells than wild-type worms and showed significantly more rapid habituation. Thus, the rate of habituation might, in part, be regulated by the amount of neurotransmitter available in the presynaptic neurons of a response circuit.

Together, the behavioral observations and the results of the GFP imaging show that the final level of connectivity of this synapse is determined by its activity during development. The evidence suggests the hypothesis that the strength of the connection between two cells is first changed by altering the number of glutamate receptors in the postsynaptic cell and then, with increased stimulation, by altering the number of vesicles in the presynaptic cell. This is consistent with observations made studying the same synapse in adult worms. Long-term habituation is mediated by a decrease in GLR-1::GFP, but there was no decrease in SNB-1::GFP in the sensory neurons unless much higher levels of training were given (Rose et al. 2003). Interestingly, our results contrast with results by Grunwald et al. (2004) who found that in C. elegans a genetic mutation that led to a chronic blockade in glutamate release (eat-4) led to an increase in GLR-1::GFP expression rather than a decrease. It is not clear why blocking activity genetically and limiting activity behaviorally would lead to opposite results. One possibility may be that the intact circuit behaves differently than a genetically altered circuit. For example, the tap sensory neurons have both chemical and electrical inputs onto the interneurons; it may be that in the intact circuit these inputs are integrated leading to one outcome, whereas in the genetically altered circuit there may be an imbalance that changes the way experience affects the circuit and leads to the opposite outcome. More studies are needed to determine the reasons for the contrasting findings.

The results reported here are consistent with mammalian studies that have examined the role of glutamate receptor regulation in developmental plasticity. In postnatal rodents, spontaneous activity in hippocampal slices leads to insertion of AMPA receptors into synapses that produces a lasting enhancement of synaptic strength (Zhu et al., 2000). Early visual deprivation in rats leads to experience-dependent changes in NMDA receptor composition and functioning (Philpot et al., 2001). There is mounting evidence that adult plasticity is mediated by the regulation of glutamate receptors (for review, see Malinow and Malenka, 2002) with increases and decreases in receptors leading to increases and decreases in synaptic strength. Our work has now extended this line of research by showing effects of rearing conditions at the level of the synapse by demonstrating alterations in glutamate receptor expression in intact animals in response to increases and decreases in experience across development.

The findings reported here for C. elegans parallel the types of changes seen in other, more complex organisms when they are raised with limited stimulation. Previous studies using genetic manipulations or laser ablation of sensory neurons in C. elegans had suggested that early experience altered development (Peckol et al., 1999; Schaefer et al., 2000; Fujiwara et al., 2002); however, these experiments are the first to examine the effects of manipulating the amount of experience received by intact, wild-type worms on behavior and development. Studies with mammals including humans (Diamond et al., 1966; Volkmar and Greenough, 1972; Turner and Greenough; 1985, Katz and Shatz, 1996; Bonhoeffer and Shatz, 1998; Joseph, 1999) have shown that stimulation during development alters growth and health in profound ways including decreases in neural growth. Because of the complexity of the mammalian nervous system, it is difficult to study these effects with the precision we can in C. elegans. Our results demonstrate that $C$. elegans is a useful model to discover the mechanisms by which experience, acting through the nervous system, profoundly alters the developing organism.

\section{References}

Apfield J, Kenyon C (1999) Regulation of life-span by sensory perception in Caenorhabditis elegans. Nature 402:804-809.

Avery L (1993) The genetics of feeding in Caenorhabditis elegans. Genetics 133:897-917.

Bellocchio E, Reimer R, Fremeau R, Edwards R (2000) Uptake of glutamate into synaptic vesicles by an inorganic phosphate transporter. Science 289:957-960.

Bonhoeffer T, Shatz CJ (1998) Neurotrophins and visual system plasticity. In: Mechanistic relationships between development and learning (Carew TJ, Menzel R, Shatz CJ, eds), pp 93-112. Chichester, UK: Wiley.

Brenner S (1974) The genetics of Caenorhabditis elegans. Genetics 77:71-94. Brockie P, Madsen D, Zheng Y, Mellem J, Maricq A (2001) Differential expression of glutamate receptor subunits in the nervous system of $C$. elegans and their regulation by the homeodomain protein UNC-42. J Neurosci 21:1510-1522.

Byerly L, Cassada RC, Russell RL (1976) The life cycle of the nematode C. elegans. I. Wild type growth and reproduction. Dev Biol 51:23-33.

Chiba C, Rankin CH (1990) A developmental analysis of spontaneous and reflexive reversals in the nematode Caenorhabditis elegans. J Neurobiol 21:543-554.

Dent JA, Davis MW, Avery L (1997) avr-15 encodes a chloride channel subunit that mediates inhibitory glutamatergic neurotransmission and ivermectin sensitivity in Caenorhabditis elegans. EMBO J 16:5867-5879.

Diamond M, Law F, Rhodes H, Lindner B, Rosenzweig M, Krech D, Bennett E (1966) Increases in cortical depth and glia numbers in rats subjected to enriched environment. J Comp Neurol 128:117-126.

Driscoll MA, Chalfie M (1991) The mec-4 gene is a member of a family of Caenorhabditis elegans genes that can mutate to induce neuronal degeneration. Nature 349:588-593.

Dusenbery D, Barr J (1980) Thermal limits and chemotaxis in mutants of the nematode C. elegans defective in thermotaxis. J Comp Physiol [A] 137:93-96.

Fujiwara M, Sengupta P, McIntire SL (2002) Regulation of body size and behavioral state of C. elegans by sensory perception and the EGL-4 cGMPdependent protein kinase. Neuron 36:1091-1102.

Grunwald ME, Mellem JE, Strutz N, Maricq AV, Kaplan JM (2004) Clathrin-mediated endocytosis is required for compensatory regulation of GLR-1 glutamate receptors after activity blockade. Proc Natl Acad Sci USA 101:3190-3195.

Hall D, Russell R (1991) The posterior nervous system of the nematode Caenorhabditis elegans: serial reconstruction of identified neurons and complete pattern of synaptic interactions. J Neurosci 11:1-22.

Hart A, Sims S, Kaplan J (1995) Synaptic code for sensory modalities revealed by C. elegans GLR-1 glutamate receptor. Nature 378:82-85.

Heisenberg M, Heusipp M, Wanke C (1995) Structural plasticity in the Drosophila brain. J Neurosci 15:1951-1960. 
Hirose T, Nakano Y, Nagamatsu Y, Misumi T, Ohta H, Oshima Y (2003) Cyclic GMP-dependent protein kinase EGL-4 controls body size and lifespan in C. elegans. Development 130:1089-1099.

Joseph R (1999) Environmental influences on neural plasticity, the limbic system, emotional development and attachment: a review. Child Psychiatry Hum Dev 29:189-208.

Katz LC, Shatz CJ (1996) Synaptic activity and the construction of cortical circuits. Science 247:1133-1138.

Lee R, Sawin E, Chalfie M, Horvitz H, Avery L (1999) EAT-4, a homolog of a mammalian sodium-dependent inorganic phosphate co-transporter, is necessary for glutamatergic neurotransmission in Caenorhabditis elegans. J Neurosci 19:159-167.

Malinow R, Malenka RC (2002) AMPA receptor trafficking and synaptic plasticity. Annu Rev Neurosci 25:103-126.

Maricq AV, Peekol E, Driscoll M, Bargmann CI (1995) Mechanosensory signaling in C. elegans mediated by the GLR-1 glutamate receptor. Nature $378: 78-81$

Nonet M (1999) Visualization of synaptic specializations in live C. elegans with synaptic vesicle protein-GFP fusions. J Neurosci Methods 89:33-40.

Peckol EL, Zallen JA, Yarrow JC, Bargmann CI (1999) Sensory activity affects sensory axon development in C. elegans. Development 126:1891-1902.

Philpot BD, Sekhar AK, Shouval HZ, Bear MF (2001) Visual experience and deprivation bidirectionally modify the composition and function of NMDA receptors in visual cortex. Neuron 29:157-169.

Rankin CH, Wicks SR (2000) Mutations of the C. elegans brain-specific inorganic phosphate transporter, eat-4, affect habituation of the tapwithdrawal response without affecting the response itself. J Neurosci 20:4337-4344.

Rankin CH, Beck CDO, Chiba C (1990) Caenorhabditis elegans: a new model system for the study of learning and memory. Behav Brain Res 37:89-92.

Rollenhagen A, Bischof HJ (1994) Spine morphology of neurons in the avian forebrain is affected by rearing conditions. Behav Neural Bio 62:83-89.

Rongo C, Kaplan JM (1999) CaMKII regulates the density of central glutamatergic synapses in vivo. Nature 402:195-199.

Rose JK, Rankin CH (2001) Analyses of habituation in C. elegans. Learn Mem 8:63-69.

Rose JK, Kaun KR, Chen SH, Rankin CH (2003) Glutamate receptor trafficking underlies long-term memory in C. elegans. J Neurosci 23:9595-9600.

Schaefer A, Hadwiger G, Nonet M (2000) rpm-1, a conserved neuronal gene that regulates targeting and synaptogenesis in C. elegans. Neuron 26:345-356.

Turner A, Greenough W (1985) Differential rearing effects on rat visual cortex synapses. Brain Res 329:195-203.

Volkmar F, Greenough W (1972) Rearing complexity affects branching of dendrites in the visual cortex of the rat. Science 176:1145-1147.

White JB, Southgate E, Thomson JN, Brenner S (1986) The structure of the nervous system of Caenorhabditis elegans. Philos Trans R Soc Lond B Biol Sci 314:1-340.

Wicks SR, Rankin CH (1995) Integration of mechanosensory stimuli in Caenorhabditis elegans. J Neurosci 15:2434-2444.

Wicks SR, Rankin CH (1997) The effects of tap withdrawal response habituation on other withdrawal behaviors: the localization of habituation in C. elegans. Behav Neurosci 111:342-353.

Zhu JI, Esteban JA, Hayashi Y, Malinow R (2000) Postnatal synaptic potentiation: delivery of GluR4-containing AMPA receptors by spontaneous activity. Nat Neurosci 3:1098-1106. 